\title{
Counselling issues in familial hypertrophic cardiomyopathy
}

B Yu, J A French, R W Jeremy, P French, D R McTaggart, M R Nicholson, C Semsarian, D R Richmond, R J Trent

Department of

Molecular and Clinical Genetics, Royal Prince Alfred Hospital, Missenden Road, Sydney, NSW 2050, Australia

B Yu

R J Trent

Department of Cardiology, Royal

Prince Alfred Hospital, Sydney, Australia

J A French

$R$ W Jeremy

C Semsarian

D R Richmond

Division of Medicine, Calvary Hospital, Canberra, Australia P French

Department of Medicine, Launceston General Hospital, Launceston, Australia D R McTaggart

Division of Cardiology, Royal Hobart Hospital, Hobart, Australia M R Nicholson

Correspondence to: Professor Trent.

Received 7 May 1997 Revised version accepted for publication 5 September publicati
1997

\begin{abstract}
To illustrate the variable clinical presentations and rates of progression in familial hypertrophic cardiomyopathy (FHC), phenotypes and genotypes were compared in three FHC families with different genetic defects. In the first family, the FHC abnormality was a protein truncating mutation (Gln969X) in the cardiac myosin binding protein $C$ gene. The second family had a missense change (Asn755Lys) in the same gene. A missense mutation (Arg453Cys) in the cardiac $\beta$ myosin heavy chain gene was present in the third family. Penetrance associated with the Gln969X defect was $27 \%$ in the age range 0 to 40 years. This was considerably less than the $93 \%$ penetrance ( 0 to 40 years) observed in the two families with missense mutations. The variable penetrance in FHC, as well as the unpredictability of sudden cardiac death, complicates clinical diagnosis and management, including genetic counselling. Although a genetic disease with a predominantly adult onset, there are counselling issues in FHC which set it aside from other adult onset disorders.

$(\mathcal{F}$ Med Genet 1998;35:183-188)
\end{abstract}

Keywords: familial hypertrophic cardiomyopathy; counselling; penetrance

The clinical spectrum found in familial hypertrophic cardiomyopathy (FHC) ranges from an asymptomatic condition to one which is associated with sudden death. The latter can occur at any age, but is more likely in children or young adults, who may also be asympto- matic. FHC is the commonest cause of sudden cardiac death in people aged $<35$ years, including those who are undertaking competitive sports. ${ }^{12}$ In one study, 48 of 134 (36\%) athletes with a cardiovascular cause for sudden death were shown at necropsy to have FHC. ${ }^{2}$ Other clinical consequences of this disorder include syncope, myocardial ischaemia, and cardiac failure. At the cellular level, a characteristic finding is extensive myocyte and myofibrillar disarray.

The prevalence of FHC ranges from 1-10 in 5000 . It is not surprising that the prevalence figure varies, since cases may remain undiagnosed using standard clinical criteria and family screening of at risk subjects is often incomplete. ${ }^{3-6}$ Traditional clinical assessment of people at risk for FHC includes medical history, physical examination, ECG, and echocardiographic measurement of ventricular wall thickness. ${ }^{56} \mathrm{~A}$ number of problems makes clinical assessment difficult, particularly in the young in whom ventricular hypertrophy may not yet have developed. Diagnostic dilemmas reflect: (1) the variable age of presentation and severity of this disorder, even within the same family, (2) investigator variance in terms of echocardiographic interpretation and measurements, and (3) the possibility that FHC represents a multifactorial or polygenic disorder in which the environment or other interacting genes play a role in pathogenesis. ${ }^{37}$

DNA studies have identified mutations in a number of muscle sarcomere genes. ${ }^{38-10}$ Following on from this, a molecular classification has been proposed to assist in the understanding of pathogenesis and to identify determinants which might influence prognosis in this condition (table 1). ${ }^{3-11}$ Despite the impressive

Table 1 Molecular classification of FHC

\begin{tabular}{llll}
\hline Type & Chromosome & Gene & Details* \\
\hline CMH1 & $14 \mathrm{q}^{*} 1$ & Cardiac $\beta$ myosin heavy chain & $>41$ mutations \\
CMH2 & $1 \mathrm{q} 3$ & Cardiac troponin T & $\sim 12$ mutations \\
CMH3 & $15 \mathrm{q} 2$ & $\alpha$ tropomyosin & $\sim 4$ mutations \\
CMH4 & $11 \mathrm{p} 11$ & Cardiac myosin binding protein C & 9 mutations, plus 2 from present study \\
CMH5 & - & - & No known locus \\
CMH6 & $7 \mathrm{q} 3$ & Not identified & Associated with Wolf-Parkinson-White syndrome \\
- & $19 \mathrm{p} 13.2-\mathrm{q} 13.2$ & Cardiac troponin I & 5 mutations \\
- & $3 \mathrm{p}$ and 12q & Myosin light chains & Rare form of FHC ( -3 mutations $)$
\end{tabular}

*The total numbers of mutations were estimated from multiple sources. 


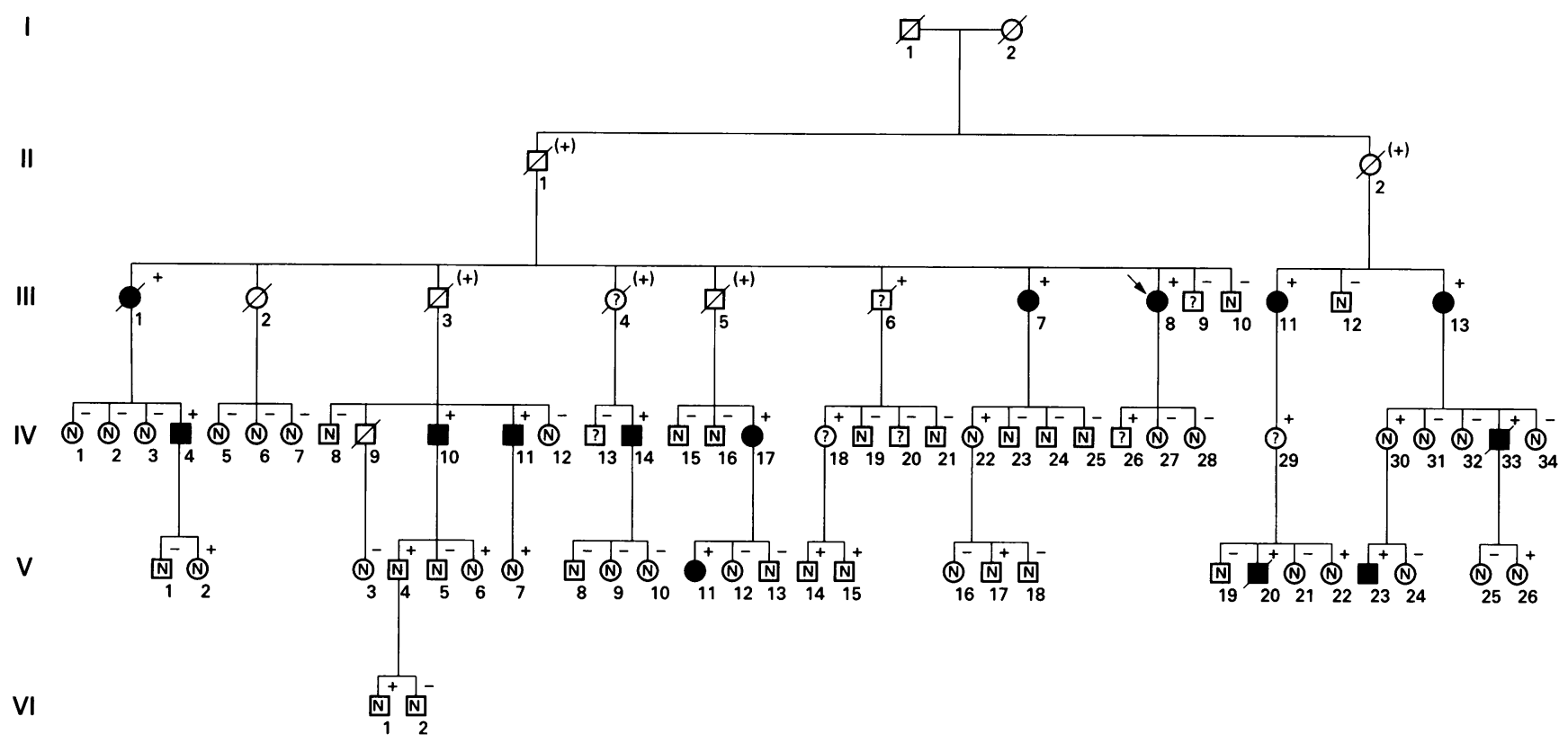

Figure 1 Pedigree of family F237.

advances in knowledge of FHC at the molecular level, the practical implications of this information have been limited by the considerable molecular heterogeneity found. This reflects both the number of genes involved and the multiplicity of mutations, with relatively few mutations recurring in unrelated families.

I

II
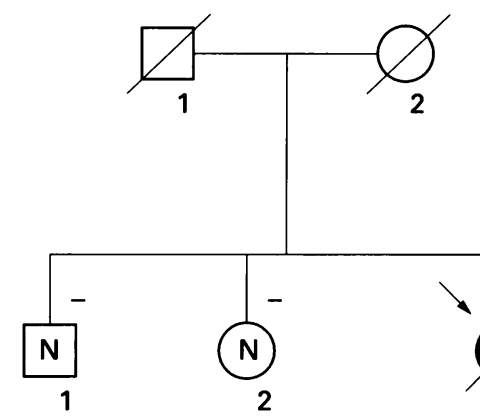

III

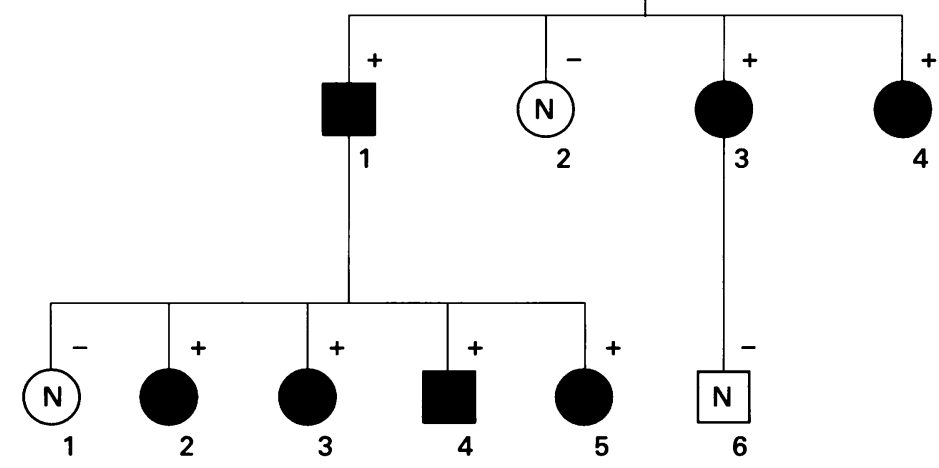

Figure 2 Pedigree of family F59 (same symbols as for fig 1).
Diagnosis of FHC and assessment of clinical outcomes remain problematical. The present study examines these difficulties with reference to three FHC families and identifies counselling issues which need careful consideration.

\section{Methods}

CLINICAL ASSESSMENT

Family F237

Sixty-nine at risk family members were studied. Clinical assessment included a medical history, physical examination, a 12 lead ECG, and echocardiogram. All clinical data were reviewed by two cardiologists independent of genetic information. A definitive diagnosis of FHC was established by echocardiographic evidence of left ventricular hypertrophy, in the absence of other known causes of myocardial hypertrophy. Criteria for hypertrophy were left ventricular wall thickness involving either the interventricular septum or the posterior wall $\geqslant 13 \mathrm{~mm}$ in adults ${ }^{56}$ or greater than the normal upper limit adjusted by body weight and body surface area in children. ${ }^{12}$ An equivocal diagnosis of FHC was made if the ventricular wall thickness was abnormal but $<13 \mathrm{~mm}$ or there was an abnormal ECG consistent with FHC, that is, left ventricular hypertrophy, abnormal $Q$ waves, or ST-T wave changes. Necropsy results were available for two additional family members who died suddenly (IV.33 and V.20) (fig 1).

\section{Families F155 and F59}

Clinical assessment for 24 at risk subjects (12 in each family) was as described above. Information for one additional member of each 
family who died from FHC (F155: IV.7, F59: II.3) was obtained from medical records. The pedigree for family F155 is found in reference 8 and that for family F59 in fig 2 .

\section{DNA MUTATION ANALYSIS}

For the cardiac myosin binding protein $\mathrm{C}$ gene (MYBPC3), mutation testing followed a standard approach involving screening of individual exons by SSCP and then DNA sequencing. More specific details of the methodology are provided in reference 8. DNA mutation analysis in family F59 was obtained by screening for common mutations in the cardiac $\beta$ myosin heavy chain gene. ${ }^{13}$

PENETRANCE DETERMINATION

Penetrance values were calculated by taking the percentage of clinically affected over the total number of DNA mutation positive subjects.

\section{Results}

CLINICAL

Family F237

The FHC phenotype in this family is generally one of a milder clinical disorder. Subjects III.1 and III.4 lived to 90 and 81 years of age respectively, while III. 11 is alive and well aged 83 years. On the other hand, two of 31 mutation positive subjects died suddenly from FHC during or following exercise. They were aged 30 (IV.33) and 7 (V.20) years (table 2). The latter had two earlier cardiac arrests associated with exercise. IV.17 underwent myomectomy because she had symptoms which were unrelieved by medical treatment. Three subjects (III.9, IV.13, IV.20) were considered equivocal on clinical criteria, but were negative on mutation testing (fig 1 ). Five others considered equivocal clinically (III.4, III.6, IV.18, IV.26, and IV.29) were subsequently shown to be mutation positive. One of the sudden

Table 2 Subjects in the three families who are DNA mutation positive and one additional obligatory carrier per family

\begin{tabular}{|c|c|c|c|c|c|c|}
\hline \multirow{2}{*}{$\begin{array}{l}\text { Pedigree } \\
\text { No }\end{array}$} & \multirow{2}{*}{$\begin{array}{l}\text { Age at } \\
\text { diagnosis* }\end{array}$} & \multirow[b]{2}{*}{$E C G+$} & \multicolumn{2}{|c|}{ Echocardiography } & \multirow{2}{*}{$\begin{array}{l}\text { Clinical } \\
\text { diagnosisf }\end{array}$} & \multirow[b]{2}{*}{ Comment $\|$} \\
\hline & & & IVS mm & $P W m m$ & & \\
\hline \multicolumn{7}{|c|}{ Family F237 } \\
\hline III. 1 & 89 & 1 & 17 & 10 & 2 & Died aged 90 y \\
\hline III.4 & 79 & 1 & 10 & 10 & 1 & Died 81 y (obligatory carrier) \\
\hline III. 6 & 74 & 1 & 12 & 8 & 1 & Died 78 y \\
\hline III.7 & 74 & Paced & 23 & 13 & 2 & \\
\hline III. 8 & 72 & 1 & 21 & 15 & 2 & \\
\hline III.11 & 83 & 1 & 22 & 16 & 2 & \\
\hline III.13 & 70 & 1 & 23 & 11 & 2 & \\
\hline IV.4 & 50 & 1 & 15 & 10 & 2 & \\
\hline IV.10 & 48 & 1 & 17 & 12 & 2 & \\
\hline IV.11 & 47 & 1 & 18 & 10 & 2 & \\
\hline IV.14 & 49 & 1 & 21 & 14 & 2 & \\
\hline IV.17 & 49 & 1 & 23 & 11 & 2 & Myomectomy 43 y \\
\hline IV.18 & 51 & 1 & 11 & 10 & 1 & \\
\hline IV.22 & 40 & 0 & 9 & 8 & 0 & \\
\hline IV.26 & 50 & 1 & 10 & 10 & 1 & \\
\hline IV.29 & 51 & 0 & 12 & 8 & 1 & \\
\hline IV.30 & 46 & 0 & 7 & 9 & 0 & \\
\hline IV.33 & 30 & NA & 40 & 20 & 2 & SCD $30 \mathrm{y}$, wall measurements made at necropsy \\
\hline V.2 & 21 & 0 & 10 & 8 & 0 & \\
\hline V.4 & 30 & 0 & 11 & 10 & 0 & \\
\hline V.6 & 24 & 0 & 11 & 9 & 0 & \\
\hline V.7 & 28 & 0 & 8 & 7 & 0 & \\
\hline V.11 & 29 & 0 & 15 & 10 & 2 & \\
\hline V.14 & 21 & 0 & 8 & 8 & 0 & \\
\hline V.15 & 19 & 0 & 9 & 6 & 0 & \\
\hline V.17 & 18 & 0 & 10 & 10 & 0 & \\
\hline V.20 & 7 & NA & NA & NA & 2 & SCD 7 y, Dx confirmed at necropsy \\
\hline V.22 & 10 & 0 & 5 & 5 & 0 & $\mathrm{NR}=5-8 \mathrm{~mm}$ \\
\hline V.23 & 20 & 1 & 15 & 10 & 2 & \\
\hline V.26 & 9 & 0 & 5 & 6 & 0 & $\mathrm{NR}=6-8 \mathrm{~mm}$ \\
\hline VI.2 & 3 & NA & 5 & 5 & 0 & $\mathrm{NR}=5-7 \mathrm{~mm}$ \\
\hline \multicolumn{7}{|c|}{ Family F155 } \\
\hline IV.1 & 39 & 1 & 25 & 10 & 2 & \\
\hline IV.3 & 37 & 1 & 12 & 11 & 1 & \\
\hline IV.5 & 32 & 1 & 20 & 11 & 2 & \\
\hline IV.7 & 19 & 1 & 29 & 29 & 2 & SCD 19 y (obligatory carrier) \\
\hline V.1 & 15 & 1 & 10 & 9 & 2 & $\mathrm{NR}=6-8 \mathrm{~mm}$ \\
\hline V.2 & 10 & 1 & 23 & 11 & 2 & $\mathrm{NR}=6-8 \mathrm{~mm}$ \\
\hline V.3 & 9 & 1 & 9 & 8 & 2 & $\mathrm{NR}=6-8 \mathrm{~mm}$ \\
\hline V.4 & 6 & 1 & 8 & 6 & 2 & $\mathrm{NR}=4-6 \mathrm{~mm}$ \\
\hline \multirow{2}{*}{\multicolumn{7}{|c|}{ Family F59 }} \\
\hline & & & & & & \\
\hline II. 3 & 50 & 1 & 15 & NA & 2 & Died 53 y (obligatory carrier) \\
\hline III. 1 & 46 & 1 & 23 & 13 & 2 & \\
\hline III. 3 & 36 & 1 & 20 & 10 & 2 & \\
\hline III.4 & 32 & 1 & 13 & 8 & 2 & \\
\hline IV.2 & 23 & 1 & 19 & 10 & 2 & \\
\hline IV. 3 & 21 & 1 & 30 & 7 & 2 & \\
\hline IV.4 & 15 & 1 & 18 & 11 & 2 & \\
\hline IV.5 & 10 & 1 & 25 & 7 & 2 & \\
\hline
\end{tabular}

*Age at diagnosis, where clinical data are available for verification or DNA testing performed if phenotypically normal. †ECG: 0 - normal, 1 - abnormal, NA - not available.

†Echocardiography: IVS - maximal interventricular wall thickness, $\mathrm{PW}$ - maximal posterior wall thickness.

SClinical diagnosis: 0 - unaffected, 1 - equivocal, 2 - affected.

ףComment: Dx - diagnosis; SCD - sudden cardiac death; NR - normal range for maximal left ventricular wall thickness. 
cardiac deaths described earlier (V.20) was the offspring of an equivocal case (IV.29).

\section{Family F155}

Except for III.1, clinical information for 13 family members extending over four generations was available (fig 1 in reference 8). Eight subjects (IV.1, 5, 7 and V.1, 2, 3, 4, 5) satisfied the clinical criteria for FHC. IV.3 was considered equivocal since the interventricular wall septum thickness was marginal and his ECG was abnormal (table 2). One of the nine mutation carriers died suddenly. IV.7 was diagnosed when aged 11 during a routine school examination and died suddenly at 19 years from ventricular fibrillation following exercise. At the time of family screening, significant septal hypertrophy was found in V.2 and V.5 when both were aged 3 years. In three children (V.3, $4,5)$, the left ventricular wall thickness increased by an unexpected $2-3 \mathrm{~mm}$ during a 1.5 year follow up period.

\section{Family F59}

The clinical picture in this family is similar to family F155, except there have been no sudden cardiac deaths to date. Significant hypertrophy was observed in affected children less than 16 years of age. II. 3 had congestive cardiac failure from the age of 50, and died three years later from embolic complications (table 2).

\section{PENETRANCE}

Truncating mutation

Thirty members of family F237 were heterozygous for the Gln969X nonsense mutation in the MYBPC3 gene. There was one obligatory carrier. Of the 31 subjects, 14 were clinically affected and five were equivocal. The penetrance in family F237 is $25 \%$ in the $0-16$ age group, increasing to $27 \%$ in the $17-40$ age group, $60 \%$ in the $41-70$ age group, and $67 \%$ in the 71 and over age group (table 2, fig 3A). The inclusion of the five equivocal cases would only change the penetrance in the latter two age groups.

\section{Missense mutations}

Eight members of family F155 were heterozygous for the Asn755Lys mutation in the MYBPC3 gene. There was one obligatory carrier. Eight of the nine were clinically affected and one was equivocal (penetrance $89 \%$ by the age of 40). Seven members of family F59 were heterozygous for the Arg453Cys mutation in the cardiac $\beta$ myosin heavy chain gene. There was one obligatory carrier. All were clinically affected (penetrance $100 \%$ by the age of 40 ). Pooling data from the two families with a missense mutation, the penetrance in the $0-40$ age group (total number of subjects $=15$ ) is $93 \%$ and $100 \%$ if the one equivocal case is included (table 2, fig 3B).

\section{Discussion}

Effective counselling in a family with FHC is dependent on an accurate diagnosis and an assessment of disease progression including the risk of sudden death. The current clinical diagnostic criteria rely on echocardiographic evidence of left ventricular hypertrophy, while
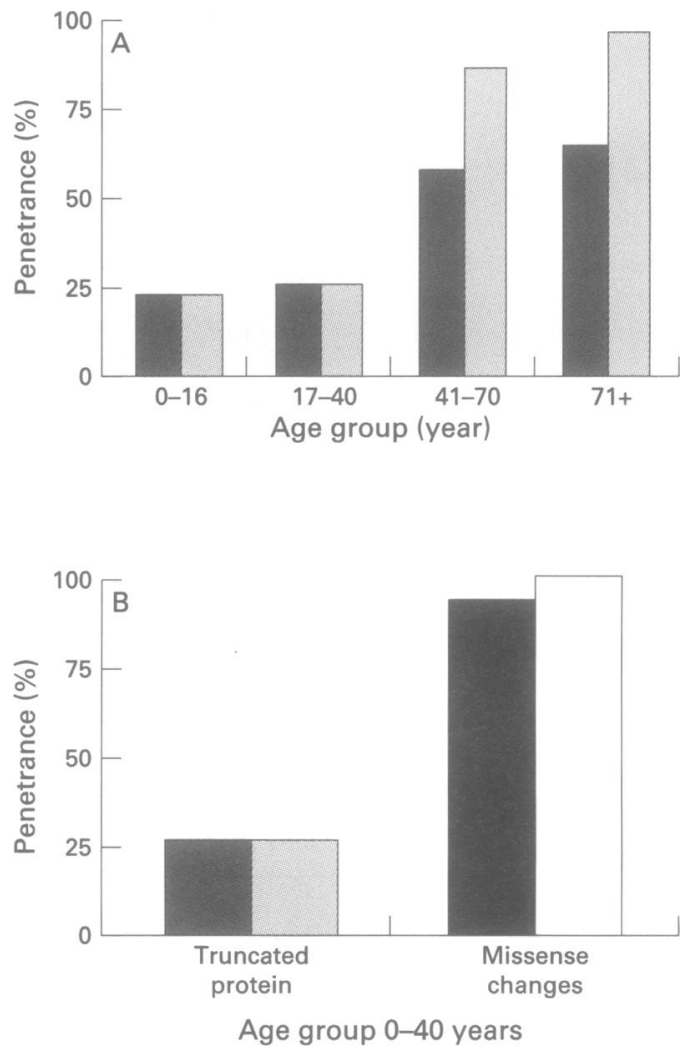

Figure 3 Penetrance in relation to age and mutation. (A) Family F237. The clinical status was determined in two ways: (1) dark grey bars represent subjects with a definite diagnosis of FHC. Numbers tested were: 0-16 age group (four subjects); 17-40 (11 subjects); 41-70 (10 subjects); $71+$ (six subjects). (2) Light grey bars represent subjects from group 1 plus cases with an equivocal $F H C$ diagnosis. (B) Comparison of penetrance in the two different types of mutations. Penetrance values are given for the MYBPC3 Gln $969 X$ truncating defect in family F237. The results are based on four of 15 mutation carriers being clinically affected in the 0-40 age group (dark grey bar). There were no equivocal cases in this group (light grey bar). Penetrance values for the two missense changes (Asn755Lys in the MYBPC3 gene and Arg453Cys in the cardiac $\beta$ myosin heavy chain gene) are based on 14 of 15 mutation positive subjects being clinically affected in the 0-40 age range (solid bar) rising to 15 of 15 if one clinically equivocal subject is included (open bar).

certain ECG abnormalities, in the absence of definitive left ventricular hypertrophy on echocardiography, are suspicious of FHC in an at risk subject. ${ }^{56}$ However, as illustrated by families F237 and F155, echocardiography is not always definitive. Equivocal cases, which are subsequently confirmed or excluded by DNA mutation analysis, are likely. Before the molecular era, conventional clinical screening approaches were considered to be deficient in children and young adults because the development of hypertrophy could be delayed. This time frame can now be extended to middle age, since the MYBPC3 defect in family F237 was not consistently detectable at this time. In this circumstance, the finding of a gene specific mutation would assist the counselling process by identifying those who remain at risk despite a normal phenotype. On the other hand, the exclusion of a DNA mutation would remove the uncertainty, especially in those who have an equivocal clinical diagnosis, a situation which affected three members of family F237. The availability of more objective data from DNA mutation analysis has highlighted the inad- 
equacy of clinical screening, with up to $20 \%$ of adults who carry a mutation shown to be clinically normal. This has prompted a review of diagnostic clinical criteria and the proposal of both major and minor ones in an attempt to increase the clinical detection rate. ${ }^{14}$

Penetrance calculations in the three families studied confirm that the clinical heterogeneity associated with FHC is considerable. The calculation of penetrance can be difficult. In FHC, this is related to the subjective nature of the clinical criteria and, in the present study, its cross sectional nature. In the case of family F155, the identification of a recently acquired de novo mutation ${ }^{8}$ has meant that the number of generations available for study is limited. Nevertheless, in family F237, it is apparent that the natural history of FHC, in terms of disease progression as measured by penetrance, is that of a milder disorder. The findings of DNA heterogeneity in FHC have raised the possibility that different DNA defects might be associated with specific clinical manifestations of this disorder. Some mutations in the cardiac $\beta$ myosin heavy chain gene (for example, Arg403Gln) have been reported to have a high association with sudden death, while others (for example, Val606Met) a low risk of death. This has given rise to the concept of "benign" and "malignant" mutations. ${ }^{715}$ However, the reliability of this classification has been questioned with a number of exceptions now observed. ${ }^{16}$ Based on sudden cardiac death, the Gln969X mutation in family F237 might be considered "malignant", yet others in the family have experienced minimal clinical consequences of this disorder. Explanations for clinical variability have also been related to the underlying protein abnormality, for example, truncated proteins (the Gln969X mutation found in family F237) can produce their effect through a reduction in the net amount of functional protein (haploinsufficiency). Missense changes (the Asn755Lys and Arg453Cys mutations) can function in autosomal dominant disorders through a dominant negative mechanism. In the three families studied, more complete penetrance was observed in two missense mutations compared to the one truncating defect. However, as stated previously, phenotype/genotype correlations in the more extensively characterised missense changes have not produced definitive trends to date. Thus, at the present time, it is not possible to correlate the underlying mutation with the clinical outcome, particularly in respect to hypertrophy and progression of disease. More data are required before the use of DNA mutation analysis, in this aspect of patient management, can be assessed.

The distinctive pathological changes which occur in FHC include ventricular hypertrophy and myocyte and myofibrillar disarray. Cellular disarray may be a critical factor in sudden cardiac death resulting from a ventricular tachyarrhythmia. There is discrepancy between the severity of hypertrophy and cellular disarray. Subjects with ventricular tachyarrhythmias do not always have obvious ventricular hypertrophy. ${ }^{4}{ }^{17}{ }^{18}$ Another explanation for heterogeneity is that FHC is a multifactorial or polygenic disorder, with the full phenotypic expression reliant on the interaction of the environment or other genes or both. ${ }^{37}$ Results from the present study are consistent with FHC being a complex genetic disorder, since all three families show considerable clinical heterogeneity despite the same DNA mutation being shared among family members.

Considerable debate has occurred in respect to the DNA testing of children for adult onset disorders, including FHC, although there does not appear to be any objection to screening children with the conventional clinical means. ${ }^{19} 2021$ However, since the clinical approaches are not always helpful, even in adults, it is not surprising that alternative diagnostic tests are in demand. Unlike other adult onset disorders, such as Huntington's disease or familial adenomatous polyposis, children with FHC can develop life threatening complications, even though their parents are normal or mildly affected. This is illustrated in family F237, with the sudden death of an offspring of a parent whose FHC clinical status was equivocal. Under these circumstances, the issues of discrimination and the effect that DNA test results may have on a child's subsequent development need to be balanced with the potential serious clinical outcomes if all available information, including DNA mutation analysis, is not obtained.

In practical terms, the molecular heterogeneity found in FHC means that DNA testing is difficult and, with current technology, not useful in the majority of families. ${ }^{13}$ However, for those families in which a mutation can be found, it is possible to exclude from subsequent follow up a number of people. Resources can then be focused on those who remain at risk. Although the association of exercise with sudden death is well known in FHC and occurred in families F237 and F155, it is relevant to note that at present there are no completely reliable clinical parameters which accurately predict this fatal complication. ${ }^{34}$ Nevertheless, a child who is shown to be DNA mutation positive may benefit from the avoidance of competitive sports and certain medications, such as the sympathomimetics, which might increase the risk of sudden death. The child would also warrant closer medical supervision and follow up. This is particularly relevant now that treatment with implantable defibrillators is available for those who have ventricular tachyarrythmias. In the context of a research proposal, the potential to follow prospectively children who are mutation positive is important to understand the natural history of FHC. In view of the complexities found in FHC, it is essential that a team approach (geneticist, counsellor, cardiologist, and molecular geneticist) is adopted in its clinical management, so that a comprehensive and effective counselling service can be offered.

This work was supported by grants from the NHMRC of Australia, the Rotary Australia Health Research Fund, and the Rebecca L Cooper Medical Research Foundation. B Yu is a recipient of a Dora Lush (Biomedical) Postgraduate Scholarship from the NHMRC. The work would not have been possible without the help and cooperation of families F237, F155, and F59. 
1 McKenna WJ, Deanfield JE. Hypertrophic cardiomyopathy an important cause of sudden death. Arch Dis Child 1984;59:971-5.

2 Maron BJ, Shirani J, Poliac LC Mathenge R, Roberts WC, Mueller FO. Sudden death in young competitive athletes: clinical, demographic, and pathological profiles. $\mathscr{f} A M A$ 1996;276:199-204.

3 Schwartz K, Carrier L, Guicheney P, Komajda M. Molecular basis of familial cardiomyopathies. Circulation 1995;91: lar basis

4 Spirito P, Seidman CE, McKenna WJ, Maron BJ. The management of hypertrophic cardiomyopathy. $N$ Engl 7 Med agement of hypert

5 Autore C, Fragola PV, Picelli A, et al. Equivocal and borderline myocardial hypertrophy in relatives of patients with hypertrophic cardiomyopathy: possible implications in genetics of the disease. Cardiology 1988;75:348-56.

6 Watkins H, McKenna WJ, Thierfelder L, et al. Mutations in the genes for cardiac troponin $\mathrm{T}$ and $\alpha$-tropomyosin in hypertrophic cardiomyopathy. N Engl $f$ Med 1995;332. 1058-64.

7 Anan R, Greve G, Thierfelder L, et al. Prognostic implications of novel beta cardiac myosin heavy chain gene mutations that cause familial hypertrophic cardiomyopathy. $\mathcal{F}$ Clin Invest 1994;93:280-5.

8 Yu B, French JA, Carrier L, et al. Molecular pathology of familial hypertrophic cardiomyopathy caused by mutations in the cardiac myosin binding protein $\mathrm{C}$ gene. $₹$ Med Genet in the cardiac my

9 Poetter K, Jiang H, Hassanzadeh S, et al. Mutations in either the essential or regulatory light chains of myosin are associated with a rare myopathy in human heart and skeletal muscle. Nat Genet 1996;13:63-9.

10 Kimura A, Harada H, Park JE, et al. Mutations in the cardiac troponin I gene associated with hypertrophic cardiomyopathy. Nat Genet 1997;16:379-82.
11 MacRae CA, Ghaisas N, Kass S, et al. Familial hypertrophic cardiomyopathy with Wolff-Parkinson-White syndrome maps to a locus on chromosome 7q3. F Clin Invest 1995;96: 1216-20.

12 Feigenbaum H. Echocardiography. 3rd ed. Philadelphia: Lea and Febiger, 1986:624.

13 Smart RV, Yu B, Le H, et al. DNA testing in familial hypertrophic cardiomyopathy - clinical and laboratory implications. Clin Genet 1996;50:169-75.

14 McKenna WJ, Spirito P, Desnos M, Dubourg O, Komajda $M$. Experience from clinical genetics in hypertrophic cardiomyopathy: proposal for new diagnostic criteria in adult members of affected families. Heart 1997;77:130-2.

15 Watkins H, Rosenzweig A, Hwang DS, et al. Characteristics and prognostic implications of myosin missense mutations in familial hypertrophic cardiomyopathy. $N$ Engl $f \mathrm{Med}$ 1992;326:1108-14.

16 Fananapazir L, Epstein ND. Genotype-phenotype correlations in hypertrophic cardiomyopathy. Insights provided by comparisons of kindreds with distinct and identical beta-myosin heavy chain gene mutations. Circulation 1994 89:22-32.

17 Maron BJ, Kragel AH, Roberts WC. Sudden death in hypertrophic cardiomyopathy with normal left ventricular mass. Br Heart f 1990;63:308-10.

18 Davis MJ, McKenna WJ. Hypertrophic cardiomyopathy pathology and pathogenesis. Histopathology 1995;26:493 patho.

19 Harper PS, Clarke A. Should we test children for "adult" genetic diseases? Lancet 1990;335:1205-6.

20 Clarke A. The genetic testing of children 7 Med Genet Clarke A. The genc
1994;31:785-97.

21 Ryan MP, French J, al-Mahdawi S, et al. Genetic testing for familial hypertrophic cardiomyopathy in newborn infants. $B M \Im$ 1995;310:856-9. 